\title{
Quantitative assessment of fatigue damage accumulation in wavy slip metals from acoustic harmonic generation
}

\author{
By John H. Cantrell \\ NASA Langley Research Center, Mail Stop 231, Hampton, VA 23681 USA
}

(Received

\begin{abstract}
A comprehensive, analytical treatment is presented of the microelastic-plastic nonlinearities resulting from the interaction of a stress perturbation with dislocation substructures (veins and persistent slip bands) and cracks that evolve during high-cycle fatigue of wavy slip metals. The nonlinear interaction is quantified by a material (acoustic) nonlinearity parameter $\beta$ extracted from acoustic harmonic generation measurements. The contribution to $\beta$ from the substructures is obtained from the analysis of Cantrell [Cantrell, J. H., 2004, Proc. R. Soc. London A, 460, 757]. The contribution to $\beta$ from cracks is obtained by applying the Paris law for crack propagation to the Nazarov-Sutin crack nonlinearity equation [Nazarov, V. E., and Sutin, A. M., 1997, J. Acoust. Soc. Am. 102, 3349]. The nonlinearity parameter resulting from the two contributions is predicted to increase monotonically by hundreds of percent during fatigue from the virgin state to fracture. The increase in $\beta$ during the first $80-90$ percent of fatigue life is dominated by the evolution of dislocation substructures, while the last 10-20 percent is dominated by crack growth. The model is applied to the fatigue of aluminium alloy 2024-T4 in stress-controlled
\end{abstract}


loading at $276 \mathrm{MPa}$ for which experimental data are reported. The agreement between theory and experiment is excellent.

PACS numbers: 62.20.Mk; 61.72.Hh; 62.65.+k; 43.25.Ba

\section{INTRODUCTION}

Various techniques and methodologies have evolved over the last several decades that attempt to characterize nondestructively the state of metal fatigue. While many of these techniques have proven useful, no method has been able to provide an unambiguous assessment of accumulated damage at a given level of fatigue over the entire range from the virgin state to fracture. Cantrell (2004) has recently shown that self-organized substructural arrangements of dislocations formed in wavy slip metals during cyclic stress-induced fatigue produce substantial changes in the microelasticplastic nonlinearity of the material that can be quantified by a material nonlinearity parameter $\beta$ experimentally determined from acoustic harmonic generation methods. The material (acoustic) nonlinearity parameter $\beta$ for a given state of fatigue is highly dependent on the volume fractions of veins and persistent slip bands (PSBs), PSB internal stresses, dislocation loop lengths, dipole heights, and the densities of secondary dislocations in the substructures.

Nazarov and Sutin (1997) have shown that the appearance of macrocracks in the material also produces large increases in $\beta$. The Paris-Erdogan (1963) equation for crack propagation is applied in the present study to the Nazarov-Sutin (1997) crack nonlinearity equation to assess the change in $\beta$ as a function of crack growth during 
the fatigue process. The resulting expression is combined with the Cantrell (2004) substructural nonlinearity model to assess the value of $\beta$ at each stage of the fatigue process from the virgin state to fracture. The model is restricted to high cycle fatigue in metals with wavy dislocation slip and is applied to the calculations of $\beta$ for fatigued polycrystalline nickel and for aluminium alloy 2024-T4 (AA2024-T4) as a function of percent total fatigue life from the virgin state to fracture. The theoretical curve is compared to the experimental data of fatigued AA2024-T4 previously reported (Cantrell and Yost 2001).

We begin in Section 2 with a discussion of relevant dislocation substructures that evolve during the fatigue of wavy slip metals. In Section 3 the contribution to $\beta$ via the Cantrell (2004) model resulting from such substructures is considered. In Section 4 the Paris-Erdogan (1963) equation is applied to the Nazarov-Sutin (1997) crack nonlinearity equation to assess the effects of crack growth during fatigue on the value of $\beta$. Theoretical $\beta$ versus percent total fatigue life curves for polycrystalline nickel and AA2024-T4 calculated from the model are given in Section 5. The results for AA2024-T4 are compared to the experimental measurements (Cantrell and Yost 2001).

\section{SUBSTRUCTURAL EVOLUTION DURING METAL FATIGUE}

Cyclic stress-induced fatigue in metals may be divided into roughly five stages: (1) cyclic hardening/softening, (2) strain localization and microcrack nucleation, (3) propagation or coalescence of microcracks to form macrocracks, (4) macrocrack propagation, and (5) fracture. Nonlinear acoustical experiments (Cantrell and Yost 
1994, 2001, Na et al. 1996, Nazarov and Sutin 1997, and Frouin et al. 1999) indicate that each stage of the fatigue process may be characterized by a unique nonlinear relationship between an impressed stress perturbation (e.g., a sound wave) and a microelastic-plastic straining of the material. The straining is quantified by an experimentally determined nonlinearity parameter $\beta$ that increases monotonically by several hundred percent over the fatigue life. In order to gain a proper understanding of the relationship between the value of the nonlinearity parameter and the state of fatigue, it is necessary to understand analytically the contribution to the nonlinearity parameter from each of the process stages. We consider first the evolution of dislocation substructures during the fatigue process.

During fatigue, the initial cycles of alternating stress generate dislocations that accumulate on the primary glide planes of the material in the form of mutually trapped primary dislocation dipoles. Under an impressed shear stress, dislocations of opposite sign in the dipole glide in opposite directions on slip planes separated by a distance $\mathrm{h}$ called the dipole height. In pure wavy slip metals the hardening/softening stage results in the growth of a so-called vein structure formed from the accumulation of dipoles generated from the cyclic stresses (Neumann 1983). The process of mutual trapping and accumulation of dislocations continues until the vein structure is composed almost entirely of dislocation dipoles. At stresses below the endurance limit both edge and screw dislocations are trapped in dipoles. At the endurance limit, and above, only edge dislocations are left. Screw dipoles are mostly annihilated by cross-slip. The vein structure can occupy as much as 50 percent volume fraction of fatigued material at saturation. 
Saturation of the vein structure occurs at a critical value of dislocation density that results in a substructural elastic instability (Kratochvil 2001). The instability marks the end of the hardening/softening stage and leads to the transformation of some of the vein structure to a more stable persistent slip band (PSB) structure (KuhlmanWilsdorf and Laird 1980, Brown 1981). The PSBs are characterized by a periodic array of parallel walls that form ladder-like patterns. The parallel walls are composed primarily of dislocation dipoles with a density roughly twice that of the vein structure. The regions between the PSB walls are dislocation poor. Because the vein structure is so hard, most of the plastic strain amplitude imposed during cyclic loading is developed in the PSBs at this stage of the fatigue process.

Crack nucleation occurs primarily at the intersection of a PSB with a bounding surface that gives rise to a stress singularity (Brown 1981). The density of microcracks nucleated during the first $20-40$ percent of fatigue life is substantial, but the cracks do not contribute significantly to the material nonlinearity until the crack length reaches a critical value. This critical value does not typically occur for high cycle fatigue of most metals until roughly $80-90$ percent of the fatigue life is expended.

\section{MICROELASTIC-PLASTIC NONLINEARITY OF SUBSTRUCTURES}

We surmise from the above considerations that the dislocation motion in fatigued metals arises primarily from two basic dislocation configurations that serve as building blocks for the veins and PSBs formed during fatigue. The first configuration is the dislocation dipole as we have discussed. The second configuration is associated 
with the fact that individual dislocations (monopoles) and even individual dislocations comprising a dipole are generally pinned by impurities or other dislocations in the material. This results in a displacement (or bowing) of the dislocation between two pinning points under an impressed shear stress. A comprehensive derivation of the equations describing the material nonlinearity arising from the dislocation configurations and fatigue substructures formed from the configurations has been recently published by Cantrell (2004). His derivation is based on the assumption of a generalized microelastic-plastic nonlinearity associated with each of the generated substructures using monopoles and dipoles as analytical building blocks. We present here the salient features of the model.

We begin by assuming that fatigued metals possess internal stresses resulting from cyclic loading and that a longitudinal stress perturbation (e.g., a sound wave) applied to the internally stressed material produces a total longitudinal strain $\varepsilon$ composed of (1) an elastic strain contribution $\varepsilon^{\mathrm{e}},(2)$ a plastic strain contribution $\varepsilon^{\mathrm{mp}}$ resulting from the bowing of pinned dislocation monopoles, and (3) a plastic strain contribution $\varepsilon^{\mathrm{dp}}$ resulting from the motion of dislocations comprising the dipoles. For expediency, it is assumed that the polycrystalline metals considered here are quasiisotropic. Hence, the total longitudinal strain in a given region of the solid is related to the elastic and two plastic strain components as

$$
\varepsilon=\varepsilon^{e}+\varepsilon^{m p}+\varepsilon^{d p} .
$$




\subsection{Elastic nonlinearity}

For elastic strains the relationship between an impressed longitudinal stress and the longitudinal elastic strain $\varepsilon^{\mathrm{e}}$ is given as (Cantrell 2004)

$$
\sigma=\sigma_{0}+A_{2}^{e} \varepsilon^{e}+\frac{1}{2} A_{3}^{e}\left(\varepsilon^{e}\right)^{2}+\cdots=\sigma_{0}+A_{2}^{e} \varepsilon^{e}-\frac{1}{2} A_{2}^{e} \beta^{e}\left(\varepsilon^{e}\right)^{2}+\cdots
$$

where $\mathrm{A}_{2}{ }^{\mathrm{e}}$ and $\mathrm{A}_{3}{ }^{\mathrm{e}}$ are the second and third-order Huang elastic coefficients, respectively, $\sigma_{0}$ is the initial stress, and $\beta^{\mathrm{e}}$ is the material elastic nonlinearity parameter defined by

$$
\beta^{e}=-\frac{A_{3}^{e}}{A_{2}^{e}}
$$

\subsection{Nonlinearity from dislocation monopoles}

To obtain the contribution to microelastic-plastic nonlinearity from dislocation monopoles, we consider some density $\Lambda^{\mathrm{mp}}$ of isolated single dislocations (dislocation monopoles) in the material lying in arbitrary slip planes in grains of arbitrary orientation in a polycrystalline solid. For a given dislocation a longitudinal stress $\sigma$ applied to the solid produces a shear stress $\tau$ at the site of the dislocation of magnitude $\tau=\mathrm{R} \sigma$ along its slip direction, where $\mathrm{R}$ is the Schmid or resolving factor. If the dislocation is pinned at two points a distance $2 \mathrm{~L}$ apart, the length of dislocation between the pinning points will bow out under the action of the resolved shear stress like an arched string. The dislocation line tension is taken to be $0.5 \mathrm{~Gb}^{2}$. The 
movement (bowing) of the dislocation produces a plastic shear strain $\gamma^{\mathrm{mp}}$ in the material. The strain $\gamma^{\mathrm{mp}}$ is related to the longitudinal monopole plastic strain $\varepsilon^{\mathrm{mp}}$ as $\varepsilon^{\mathrm{mp}}$ $=\Omega \gamma^{\mathrm{mp}}$ where $\Omega$ is the conversion factor from shear to longitudinal strain.

The dislocation monopole stress-strain relationship is given by (Cantrell 2004)

$$
\sigma=\sigma_{0}+A_{2}^{m p} \varepsilon^{m p}-\frac{1}{2} A_{2}^{m p} \beta^{m p}\left(\varepsilon^{m p}\right)^{2}+\cdots
$$

where

$$
A_{2}^{m p}=\frac{3}{2} \frac{G}{\Omega \Lambda^{m p} L^{2} R}
$$

and

$$
\beta^{m p}=\frac{24}{5} \frac{\Omega \Lambda^{m p} L^{4} R^{3}\left(A_{2}^{e}\right)^{2}}{G^{3} b^{2}}\left|\sigma_{0}\right|
$$

In Eqs.(5) and (6) $\mathrm{G}$ is the shear elastic modulus, $\mathrm{b}$ is the amplitude of the Burgers vector, and $\left|\sigma_{0}\right|$ is the magnitude of the initial (residual or internal) longitudinal stress in the material.

\subsection{Nonlinearity from dislocation dipoles}

The contribution from dislocation dipoles to the microelastic-plastic nonlinearity is obtained by noting that for edge dislocation pairs of opposite polarity the shear force per unit length $F_{x}$ along the glide plane on a given dislocation due to the other dislocation in the pair is given by (Hull and Bacon 1984) 


$$
F_{x}=-\frac{G b^{2}}{2 \pi(1-v)} \frac{x\left(x^{2}-y^{2}\right)}{\left(x^{2}+y^{2}\right)^{2}}
$$

In Eq.(7) $v$ is Poisson's ratio, and (x,y) are the Cartesian coordinates of one dislocation in the pair relative to the coordinates $(0,0)$ of the second. As mentioned previously, the motion of the dislocations in the dipole pair occurs only along parallel slip directions separated by the dipole height h. Using Eq.(7), Cantrell (2004) has shown that for a material having a density $\Lambda^{\mathrm{dp}}$ of dislocation dipoles, the dislocation dipole stress-plastic strain relationship is given as

$$
\sigma=\sigma_{0}+A_{2}^{d p} \bar{\varepsilon} d p-\frac{1}{2} A_{2}^{d p} \beta^{d p}\left(\bar{\varepsilon}^{d p}\right)^{2}+\cdots
$$

where

$$
A_{2}^{d p}=\left(\frac{G}{4 \pi \Omega R \Lambda^{d p} h^{2}(1-v)}\right)
$$

and

$$
\beta^{d p}=\frac{16 \pi^{2} \Omega R^{2} \Lambda^{d p} h^{3}(1-v)^{2}\left(A_{2}^{e}\right)^{2}}{G^{2} b}
$$

\subsection{Effective microelastic-plastic nonlinearity parameter}

Each of the stress-strain relationships [Eqs.(2), (4) and (8)] can be solved for the strain in terms of the stress to second order in the stress. Adding the strains together to obtain the total strain $\varepsilon$ as given by Eq.(1) and solving the resulting expression for the stress in terms of the total strain lead to the relationship for the effective nonlinearity parameter for the fatigued material given by (Cantrell 2004) 


$$
\beta=\frac{\beta^{e}+\beta^{m p}+\beta^{d p}}{\left(1+\Gamma^{m p}+\Gamma^{d p}\right)^{2}}
$$

where the gamma factors

$$
\Gamma^{m p}=\frac{2}{3}\left(\frac{\Omega \Lambda^{m p} L^{2} R}{G}\right) A_{2}^{e}
$$

and

$$
\Gamma^{d p}=\frac{4 \pi A_{2}^{e} \Omega R \Lambda^{d p} h^{2}(1-v)}{G} .
$$

\subsection{Substructural contributions to the nonlinearity parameter of wavy slip metals}

Eq.(11) is obtained under the assumption that the dislocation monopoles and dipoles are distributed uniformly throughout the material. Generally, the monopoles and dipoles are not distributed uniformly but are located within the discrete substructures generated in the material during fatigue. We denote the total volume fraction of substructure containing monopoles by $\mathrm{f}^{\mathrm{mp}}$ and the total volume fraction of substructure containing dipoles by $\mathrm{f}^{\mathrm{dp}}$. We assume that the values of the monopole and dipole nonlinearity parameters, $\beta^{\mathrm{mp}}$ and $\beta^{\mathrm{dp}}$, and gamma factors, $\Gamma^{\mathrm{mp}}$ and $\Gamma^{\mathrm{dp}}$, are constant within the fatigue-generated substructures. The change in the material nonlinearity parameter due to fatigue-generated substructures is obtained by summing the individual contributions from dislocation monopole and dislocation dipole sources weighted by the volume fractions of material making such contributions. Thus, the total nonlinearity parameter is more appropriately given as 


$$
\beta=\frac{\beta^{e}+f^{m p} \beta^{m p}+f^{d p} \beta^{d p}}{\left(1+f^{m p} \Gamma^{m p}+f^{d p} \Gamma^{d p}\right)^{2}} .
$$

The dislocation dipole contribution has a possible vein structure source and a possible PSB source depending on the state of fatigue. We thus write $\mathrm{f}^{\mathrm{dp}} \beta^{\mathrm{dp}}=\mathrm{f}_{\text {vein }} \beta_{\text {vein }}^{\mathrm{dp}}+\mathrm{f}_{\mathrm{PSBw}} \beta_{\mathrm{PSBw}}^{\mathrm{dp}}$ and $\mathrm{f}^{\mathrm{dp}} \Gamma^{\mathrm{dp}}=\mathrm{f}_{\text {vein }} \Gamma_{\text {vein }}^{\mathrm{dp}}+\mathrm{f}_{\mathrm{PSBW}} \Gamma_{\text {PSBw }}^{\mathrm{dp}}$ where $\mathrm{f}_{\text {vein }}$ and $\mathrm{f}_{\mathrm{PSBW}}$, respectively, are the volume fractions of material at a given percent full life containing vein structure and PSB wall structure. We assume $\mathrm{f}_{\mathrm{PSBw}}=(1 / 8) \mathrm{f}_{\mathrm{PSB}}$ where $\mathrm{fPSB}_{\mathrm{PB}}$ is the volume fraction of PSBs in the material and the factor (1/8) is an estimate of the fraction of material in the PSB containing wall structure (Winter 1974, Antonopoulos and Winter 1976, Antonopoulos, Brown and Winter 1976). The dislocation monopole contribution has a vein structure source and a PSB structure source resulting from the generation of secondary dislocations. It is important to note that PSBs with secondary dislocations are old ones - not freshly initiated. Hence, we write $\mathrm{f}^{\mathrm{mp}} \beta^{\mathrm{mp}}=\mathrm{f}_{\text {vein }} \beta_{\text {vein }}^{\mathrm{mp}}+\mathrm{f}_{\mathrm{PSB} \sigma} \beta_{\mathrm{PSB} \sigma}^{\mathrm{mp}}$ and $\mathrm{f}^{\mathrm{mp} p} \Gamma^{\mathrm{mp}}=\mathrm{f}_{\text {vein }} \Gamma_{\text {vein }}^{\mathrm{mp}}+\mathrm{f}_{\mathrm{PSB} \sigma} \Gamma_{\mathrm{PSB} \sigma}^{\mathrm{mp}}$ where $\mathrm{f}_{\mathrm{PSB} \sigma}$ is the volume fraction of material within the PSBs that contains sufficiently large secondary dislocation densities to significantly influence $\beta$ via the PSB internal stress field. A crude estimate of $\mathrm{fSB}_{\mathrm{PS} \sigma} \approx 0.15 \mathrm{fSSB}_{\mathrm{Pay}}$ be obtained from transmission electron micrographs of secondary dislocation structures in PSBs (Wang and Mughrabi 1984, Wang et al. 1984). Substituting these equations into Eq.(14) yields 


$$
\beta=\frac{\beta^{e}+f_{\text {vein }}\left(\beta_{\text {vein }}^{m p}+\beta_{\text {vein }}^{d p}\right)+f_{P S B}\left(0.15 \beta_{P S B \sigma}^{m p}+0.125 \beta_{P S B w}^{d p}\right)}{\left[1+f_{\text {vein }}\left(\Gamma_{\text {vein }}^{m p}+\Gamma_{\text {vein }}^{d p}\right)+f_{P S B}\left(0.15 \Gamma_{P S B \sigma}^{m p}+0.125 \Gamma_{P S B w}^{d p}\right)\right]^{2}} .
$$

\section{EFFECTS OF FATIGUE CRACKS ON NONLINEARITY PARAMETER}

The nucleation of cracks in the interior of pure polycrystalline metals is generally confined to the intersection of PSBs with bounding surfaces, such as appropriately-angled grain boundaries or (incoherent) inclusions, that can provide the appropriate conditions necessary to engender a stress singularity at the intersection (Brown 1981). Nazarov and Sutin (1997) derived an expression for the contribution to the nonlinearity parameter of non-interacting penny-shaped cracks in bulk material. Using their suggested values of crack-related constants, we write their results in terms of the present notation as

$$
\beta^{c r k} \approx \frac{5.3 \times 10^{6} C_{c r k} R_{c r k}^{4}}{\left(1+0.25 C_{c r k} R_{c r k}^{3}\right)^{2}}
$$

where $\mathrm{C}_{\text {crk }}$ is the concentration of cracks in the interior of the material and $\mathrm{R}_{\text {crk }}$ is the radius of the crack.

In order to obtain the crack contribution to the total nonlinearity parameter as a function of crack growth during the fatigue process we employ the Paris-Erdogan (1963) equation to assess the variation in the crack radius $R_{\text {crk }}$ as a function of percent fatigue life to fracture. The Paris-Erdogan (1963) equation is given as

$$
\frac{d R_{c r k}}{d N}=A(\Delta K)^{n}
$$


where $\mathrm{N}$ is the number of fatigue cycles, $\Delta \mathrm{K}$ is the stress-intensity range, and $\mathrm{A}$ and $\mathrm{n}$ are material-dependent constants. The stress-intensity range is related to $R_{\text {crk }}$ as (Hertzberg 1989)

$$
\Delta K=B \Delta \sigma \sqrt{R_{c r k}}
$$

where $\Delta \sigma=\sigma_{\max }-\sigma_{\min }$ is the difference between the maximum and minimum cyclic loading stresses and B is a factor that depends on the geometry of the crack. We substitute Eq.(18) into Eq.(17) and integrate the resulting expression with respect to $R_{\text {crk }}$ between some arbitrary crack radius $R_{\text {crk1 }}$ and the critical crack radius $R_{\text {crk2 }}$ to ascertain the number of loading cycles $\Delta \mathrm{N}$ remaining from the appearance of a crack of radius $\mathrm{R}_{\text {crk1 }}$ to fracture. We obtain

$$
\begin{gathered}
\Delta N=\frac{1}{A(B \Delta \sigma)^{n}} \int_{R_{c k k 1}}^{R_{c k 2}} R_{c r k}^{-n / 2} d R_{c r k} \\
=\frac{2}{(n-2) A(B \Delta \sigma)^{n}}\left|\frac{1}{R_{c r k 1}^{(n / 2)-1}}-\left(\frac{B \sigma_{\max }}{K_{1 c}}\right)^{n-2}\right| .
\end{gathered}
$$

The critical crack radius is defined as that radius such that further loading leads to catastrophic failure. The last term in brackets in Eq.(19) is thus obtained by setting the 
critical crack radius $\mathrm{R}_{\mathrm{crk} 2}=\left(\mathrm{K}_{1 \mathrm{c}} / \mathrm{B} \sigma_{\max }\right)^{2}$ where $\mathrm{K}_{1 \mathrm{c}}$ is the fracture toughness of the material (Hertzberg 1989).

Let $\mathrm{N}_{\text {total }}$ equal the total number of loading cycles from the virgin state to fracture. The fraction of fatigue life remaining for the material occurring after the appearance of a crack of radius $\mathrm{R}_{\text {crk1 }}$ is $\Delta \mathrm{N} / \mathrm{N}_{\text {total }}$. The crack of radius $\mathrm{R}_{\text {crk1 }}$ thus occurs at the fraction $\mathrm{f}=\left[1-\left(\Delta \mathrm{N} / \mathrm{N}_{\text {total }}\right)\right]$ of total fatigue life of the material measured from the virgin state. Setting $R_{c r k 1}=R_{c r k}$ in the last equality in Eq.(19), solving for $R_{\text {crk }}$ and substituting the resulting expression in Eq.(16), we obtain that the crack growth contribution $\beta^{\text {crk }}$ to the total nonlinearity parameter $\beta$ as a function of the fraction of total fatigue life $\mathrm{f}$ is given as

$$
\beta^{c r k}=\frac{5.3 \times 10^{6} C_{c r k}}{\left[a_{1}^{n-2}+a_{2}(1-f)\right]^{8 /(n-2)}}\left[1+\frac{0.25 C_{c r k}}{\left[a_{1}^{n-2}+a_{2}(1-f)\right]^{-6 /(n-2)}}\right]^{-2}
$$

where

$$
a_{1}=\frac{B \sigma_{\max }}{K_{1 c}}
$$

and

$$
a_{2}=\frac{(n-2)}{2} N_{\text {total }} \mathrm{A}(B \Delta \sigma)^{n}
$$

It is emphasized that Eq.(20) is applicable in the Paris law regime where $\mathrm{R}_{\mathrm{crk}}$ is of the order $250 \mu \mathrm{m}$ or larger. Such crack lengths generally occur in the final 10-20 percent 
of fatigue life for high cycle fatigue. Cracks having a radius smaller than $250 \mu \mathrm{m}$ are seen directly from Eq.(16) to make a negligible contribution to $\beta^{\text {crk }}$ for typical values of $\mathrm{C}_{\mathrm{crk}}$ [of order $10^{7} \mathrm{~m}^{-3}$ (Nazarov and Sutin 1963)].

\section{DEPENDENCE OF NONLINEARITY PARAMETERS OF NICKEL AND}

\section{AA2024-T4 ON STATE OF FATIGUE}

We consider the application of the above model to the calculation of the nonlinearity parameters of polycrystalline nickel and AA2024-T4 as a function of percent full fatigue life to fracture. Grobstein et al. (1991) published an extensive study of substructural evolution in fatigued polycrystalline nickel, a wavy slip metal, cyclically stressed from the virgin state to fracture using various specimens subjected to a variety of loading conditions. Their data include volume fractions of dislocation substructures (veins and PSBs) and dislocation densities within the substructures formed as a function of percent full life. We apply their data in Section 5.1 to the calculations of the nonlinearity parameters of polycrystalline nickel for specimens fatigued in fully reversed, stress-controlled loading conditions at $241 \mathrm{MPa}$ and at $345 \mathrm{MPa}$.

Correspondingly detailed substructural evolution data are not available for aluminium alloy 2024-T4. The relationship between the microstructure and cyclic plastic-deformation behavior is generally more complex for high strength commercial alloys than that of pure metals. It is generally known, for example, that the PSBs in such alloys are thinner and carry a much larger plastic strain than pure metals (Christ 1996). Despite the more complex nature of the dislocation substructures for AA2024- 
$\mathrm{T} 4$, it is important to emphasize that the basic building blocks of the substructures remain dislocation monopoles and dislocation dipoles. It is these basic building blocks that determine the magnitude of the nonlinearity parameters. In this view we naively assume that since AA2024-T4 is also a wavy slip metal, the nonlinearity parameters of the alloy may be obtained from the substructural evolution data of nickel Grobstein et al. (1991) and the elastic constants, Poisson ratio, and Burgers vector for AA2024-T4. We apply the above analytical model in Section 5.2 to the calculation of $\beta$ for AA2024-T4 as a function of percent full life to fracture for specimens cyclically loaded in uniaxial, stress-control at 276MPa from zero to full load. The results are compared to experimental data previously reported for AA2024-T4 (Cantrell and Yost 2001).

\subsection{Polycrystalline nickel}

\subsubsection{Stress-controlled loading at 241MPa}

For stress-controlled cyclic loading at $241 \mathrm{MPa}$ we use the dislocation substructure-related parameters previously ascertained by Cantrell (2004) for polycrystalline nickel from the data of Grobstein et al. (1991) and the published works of Winter (1974), Antonopoulos and Winter (1976), Antonopoulos, Brown and Winter (1976), Brown (1981), Wang and Mughrabi (1984) and Wang et al. (1984). The vein structure attains a volume fraction $\mathrm{f}_{\text {vein }}=0.35$ at 10 percent full life and remains at that volume fraction to fracture. PSBs begin to form at $\sim 0.1$ percent full life and monotonically grow with decreasing slope to a volume fraction $\mathrm{f}_{\mathrm{PSB}}=0.158$ at full life. The dislocation density in the PSB walls $\Lambda^{\mathrm{mp}}{ }_{\mathrm{PSBw}}=1.7 \times 10^{15} \mathrm{~m}^{-2}$, the dislocation 
density in the vein structure $\Lambda^{\mathrm{mp}}{ }_{\text {vein }}=8.5 \times 10^{14} \mathrm{~m}^{-2}$, the shear modulus $\mathrm{G}=73.2 \mathrm{GPa}$, the longitudinal modulus $\mathrm{A}_{2}{ }_{2}=278 \mathrm{GPa}$, the Poisson ratio $v=0.39$, the magnitude of the Burgers vector $b=0.249 \mathrm{~nm}$, and the dislocation half-loop length $\mathrm{L}=8.2 \times 10^{-8} \mathrm{~m}$. The dipole density in the vein structure $\Lambda_{\text {vein }}^{\mathrm{dp}}=4.25 \times 10^{14} \mathrm{~m}^{-2}$, the dipole density in the wall structure $\Lambda_{\mathrm{PSBw}}^{\mathrm{dp}}=8.5 \times 10^{14} \mathrm{~m}^{-2}$, the dipole height in the vein structure $\mathrm{h}_{\text {vein }}$ $=7.6 \mathrm{~nm}$, and the dipole height in the PSBs $\mathrm{h}_{\mathrm{PSB}}=5.4 \mathrm{~nm}$. For polycrystalline solids we assume $\Omega \approx \mathrm{R} \approx 1 / 3$.

The vein structure is characterized by short-range stresses resulting from the relaxation of the dislocation substructure into its equilibrium arrangement. The dislocation monopole contribution to the nonlinearity parameter from the vein structure $\beta_{\text {vein }}^{\mathrm{mp}}$ is obtained from Eq.(6). Mughrabi (1981) estimated that the internal (initial) shear stress $\tau$ experienced by a given dislocation in the vein structure in copper is roughly 20 percent of the saturation shear stress (28MPa for copper). According to the model of Brown (2000), the edge dislocation line tension is closer to $0.23 \mathrm{nN}$ rather than the value $1.0 \mathrm{nN}$ estimated by Mugrhabi. Thus, from Brown's model we estimate that the internal stress in the vein structure is of the order 4.6 percent of the saturation stress. For copper this means an internal shear stress $\tau_{\text {vein,Cu }}$ of $1.28 \mathrm{MPa}$. Mughrabi, Ackermann and Herz (1978) have shown that the saturation stress scales as the magnitude of the shear modulus of the material. Thus, the internal shear stress of nickel $\tau_{\mathrm{vein}, \mathrm{Ni}}$ is calculated as $\tau_{\mathrm{vein}, \mathrm{Ni}}=\left(\mathrm{G}_{\mathrm{Ni}} / \mathrm{G}_{\mathrm{Cu}}\right) \tau_{\mathrm{vein}, \mathrm{Cu}}$, where $\mathrm{G}_{\mathrm{Ni}}$ is the shear modulus of nickel and $\mathrm{G}_{\mathrm{Cu}}$ is the shear modulus of copper Since $\mathrm{G}_{\mathrm{Ni}}=73.2 \mathrm{GPa}$ and $\mathrm{G}_{\mathrm{Cu}}=$ 42.0GPa, we obtain $\tau_{\text {vein,Ni }}=2.23 \mathrm{MPa}$. The effective longitudinal stress $\left|\sigma_{0}\right|$ that appears in Eq.(6) is calculated as $\left|\sigma_{0}\right|=|\tau| / \mathrm{R}=6.69 \mathrm{MPa}$ for polycrystalline nickel. 
From Eq.(6), the dislocation monopole contribution to the nonlinearity parameter from the vein structure is calculated to be $\beta_{\text {vein }}^{\mathrm{mp}}=48.4$. The gamma factor for dislocation monopoles in the vein structure is calculated from Eq.(12) to be $\Gamma^{\mathrm{mp}}$ vein $=1.61$.

The calculation of the contribution to the nonlinearity parameter from dislocation dipoles in the vein structure is obtained by substituting the material constants and dislocation-related parameters given above into Eq.(10). We obtain $\beta_{\text {vein }}^{\mathrm{dp}}=23.5$ for the value of the dipole contribution associated with the vein structure. The gamma factor for dislocation dipoles in the vein structure is calculated from Eq.(13) to be $\Gamma^{\mathrm{dp}}{ }_{\mathrm{vein}}=0.08$.

The dipole contribution to the nonlinearity parameter associated with the PSB wall (ladder) structure is also obtained from Eq.(10) using the same values of the nickel material parameters as that for the vein structure except that for PSB walls the dislocation density $\Lambda^{\mathrm{dp}}{ }_{\mathrm{PSBw}}=8.5 \times 10^{14} \mathrm{~m}^{-2}$ and the dipole height $\mathrm{h}_{\mathrm{PSB}}=5.4 \mathrm{~nm}$. Using these values, we obtain the PSB wall dipole contribution to be $\beta_{\mathrm{PSBw}}^{\mathrm{dp}}=16.9$. The gamma factor for dislocation dipoles in the PSB wall structure is calculated from Eq.(13) to be $\Gamma^{\mathrm{dp}}{ }_{\mathrm{PSBw}}=0.08$.

A substantial contribution to the nonlinearity parameter is also obtained from the action of initial (internal) stresses on dislocations generated on secondary slip systems as the PSBs mature. According to Brown and Ogin (1981), the internal tensile stress $\sigma_{\mathrm{PSB}}$ generated in the PSBs is given as

$$
\sigma_{P S B} \approx \frac{5.7}{2 \pi(1-v)} G e_{t}
$$


where

$$
e_{t} \approx(\phi-\theta) \varepsilon_{p l}
$$

and $(\phi-\theta)$ is the misorientation of the PSB walls with respect to the matrix. Assuming a misorientation of 5 degrees (Brown and Ogin 1981) and a typical maximum plastic strain $\varepsilon_{\mathrm{pl}}$ for pure metals of roughly $7.5 \times 10^{-3}$, we obtain from Eqs.(23) and (24) that

$$
\sigma_{P S B}=5.938 \times 10^{-4} \frac{G}{(1-v)} .
$$

The tensile stress $\sigma_{\mathrm{PSB}}$ is orientated roughly along the direction of the Burgers vector. The resolved shear stress $\tau_{\text {second }}$ along the secondary slip direction is thus given by $\tau_{\text {second }}=\mathrm{R}^{\prime} \sigma_{\mathrm{PSB}}$ where $\mathrm{R}^{\prime} \approx 0.41$. The longitudinal stress $\sigma_{0}$ given in Eq.(6) is related to $\tau_{\text {second }}$ and to $\sigma_{\mathrm{PSB}}$ as $\sigma_{0}=\tau_{\text {second }} / \mathrm{R}=\left(\mathrm{R}^{\prime} / \mathrm{R}\right) \sigma_{\mathrm{PSB}}=1.23 \sigma_{\mathrm{PSB}}$. For polycrystalline nickel we thus calculate that $\left|\sigma_{0}\right|=87.5 \mathrm{MPa}$.

Finally, according to Brown (1981) the tensile stresses that develop along the slip direction in the PSBs do not interact with the dislocations in the primary slip plane. Rather, they interact with secondary dislocations. Transmission electron micrographs obtained by Wang and Mughrabi (1984) and Wang et al. (1984) suggest that the density of secondary dislocations approaches that of the PSB wall structure ( $2 \times 10^{15} \mathrm{~m}^{-2}$ ) as mature wall structures transform into misoriented cells during secondary hardening. We assume here a secondary dislocation density of $1.0 \times 10^{15}$ $\mathrm{m}^{-2}$. Using these values together with the values of the elastic constants, half-loop 
length, Burgers vector, $\mathrm{R}$, and $\Omega$ used in the preceding calculations, we calculate from Eq.(6) the contribution to the nonlinearity parameter from PSB stresses to be $\beta_{\mathrm{PSB} \sigma}^{\mathrm{mp}}=$ 745.3. The gamma factor for dislocation monopoles associated with secondary dislocations is calculated from Eq.(3.70) to be $\Gamma^{\mathrm{mp}}{ }_{\mathrm{PSB} \sigma}=1.89$.

\subsubsection{Stress-controlled loading at $345 \mathrm{MPa}$}

For stress-controlled cyclic loading conditions at $345 \mathrm{MPa}$ we use the same dislocation substructure-related parameters given above for $241 \mathrm{MPa}$ except that for $345 \mathrm{MPa}$ the fatigue parameters $\mathrm{f}_{\mathrm{vein}}=0.44, \mathrm{f}_{\mathrm{PSB}}=0.224, \Lambda^{\mathrm{mp}}{ }_{\mathrm{PSBw}}=2.1 \times 10^{15} \mathrm{~m}^{-2}$, $\Lambda_{\text {vein }}^{\mathrm{mp}}=1.05 \times 10^{15} \mathrm{~m}^{-2}, \Lambda_{\text {vein }}^{\mathrm{dp}}=5.25 \times 10^{14} \mathrm{~m}^{-2}$, and $\Lambda^{\mathrm{dp}}{ }_{\mathrm{PSBw}}=1.05 \times 10^{15} \mathrm{~m}^{-2}$. Using these parameters, we calculate $\beta_{\text {vein }}^{\mathrm{mp}}=59.8, \Gamma_{\text {vein }}^{\mathrm{mp}}=1.99, \beta_{\mathrm{vein}}^{\mathrm{dp}}=29.05$, $\Gamma_{\mathrm{vein}}^{\mathrm{dp}}=0.098, \beta_{\mathrm{PSBw}}^{\mathrm{dp}}=20.8$, and $\Gamma_{\mathrm{PSBw}}^{\mathrm{dp}}=0.099$.

\subsubsection{Polycrystalline nickel nonlinearity parameter versus percent fatigue life}

The total nonlinearity parameters $\beta$ for polycrystalline nickel resulting from substructural evolution are calculated by substituting the substructural contributions to the nonlinearity parameters and gamma factors obtained in Sections 5.1.1 and 5.1.2 into Eq.(15). The contribution to $\beta$ from crack growth is obtained from Eqs.(20)-(22). For polycrystalline nickel we assume $\mathrm{n} \approx 3.0, \mathrm{~B}=1.13$ (penny-shaped cracks), and $\mathrm{K}_{1 \mathrm{c}}$ $\approx 62 \mathrm{MPa} \sqrt{\mathrm{m}}$ (Mirshams et al. 2001). From the data of Grobstein et al. (1991) for

uniaxial, fully reversed, stress-controlled loading at $154 \mathrm{MPa}$, we obtain $\mathrm{A} \approx 8.6 \times 10^{-10}$ 
$\mathrm{MPa}^{-3} \mathrm{~m}^{-0.5}$ cycle $^{-1}$ assuming from the data that $\Delta \mathrm{N} \approx 3.3 \times 10^{3}$ cycles, $\mathrm{N}_{\text {total }} \approx 2.23 \mathrm{x}$ $10^{4}$ cycles, and $\mathrm{C}_{\mathrm{crk}} \approx 10^{7} \mathrm{~m}^{-3}$ (Nazarov and Sutin 1963 ).

The total nonlinearity parameters $\beta$ for pure polycrystalline nickel are plotted in Fig.1 as a function of percent fatigue life from the virgin state to fracture for stresscontrolled loading at $241 \mathrm{MPa}$ and for stress-controlled loading at $345 \mathrm{MPa}$. The substructural contribution to $\beta$ in the plots are obtained by substituting into Eq.(15) the substructural evolution data of Grobstein et al. (1991) and the above-calculated values of the nonlinearity parameters and gamma factors. The contributions from crack growth are calculated from Eqs.(20)-(22) and the above-calculated Paris law parameters using the data of Grobstein et al. (1991) for stress-controlled loading at $154 \mathrm{MPa}$. It is assumed that the results, when expressed in terms of percent total life, may be applied in good approximation to the cases of stress-controlled loading at $241 \mathrm{MPa}$ and $345 \mathrm{MPa}$ when the latter results are also expressed in terms of percent total life. The contribution from the elastic nonlinearity $\beta^{\mathrm{e}}$ is estimated from elastic constant data to be approximately 5.0 (Cantrell 2004).

It is evident from Fig. 1 that despite significant variations in the loading stresses and volume fractions of veins and PSBs for the two cases, the model predicts quite similar variations of the nonlinearity parameters. Both curves show monotonically increasing values of $\beta$ of roughly 300 percent over the fatigue life from substructural evolution alone. The values of the nonlinearity parameters for the two loading conditions agree to within three percent from approximately 0.01 percent total life to fracture. The larger difference in $\beta$ values below 0.01 percent results directly from the difference in the number of fatigue cycles to fracture for the two loads. A measurable 
contribution from crack growth begins roughly at 85 percent of total fatigue life and rapidly becomes the dominant contribution to $\beta$ as the crack increases in size to fracture.

\subsection{Aluminium alloy 2024-T4}

Detailed substructural evolutionary data comparable to that obtained by Grobstein et al. (1991) for polycrystalline nickel are not available for aluminium alloy 2024-T4 (AA2024-T4). However, since AA2024-T4 is a wavy slip metal, the substructural evolution of AA2024-T4 may be expected to follow a pattern somewhat similar to that of polycrystalline nickel, although the exact details of the substructural organization (e.g., dimensions, volume fractions, morphology, etc.) differ due to precipitation and alloying (Christ 1996). It is important to recognize in the present model that the more important consideration for model calculations is the dependence of the total nonlinearity parameter directly on the dislocation monopoles and dipoles as basic building blocks for the more complex fatigue-induced substructures. The model may thus be regarded as somewhat generic in character. In this view we assume that the substructural contributions to the total nonlinearity parameter as a function of percent full fatigue life may be estimated from Eq.(15) using the substructural evolution data of Grobstein et al. (1991) and from Eqs.(6), (10), (12), (13), and (25) using the material constants for AA2024-T4. For AA2024-T4 the appropriate material constants are $\mathrm{G} \approx 28.6 \mathrm{GPa}, \mathrm{A}_{2}{ }^{\mathrm{e}} \approx 109 \mathrm{GPa}, v=0.33$, and $\mathrm{b} \approx$ $0.286 \mathrm{~nm}$. The values of the half-loop length and dipole heights are assumed to be the same as that used for nickel. 
We thus obtain for AA2024-T4 that $\beta_{\text {matrix }}^{\mathrm{mp}}=37.0, \Gamma_{\text {matrix }}^{\mathrm{mp}}=1.61, \beta_{\text {matrix }}^{\mathrm{dp}}=$ 24.9, $\Gamma_{\text {matrix }}^{\mathrm{dp}}=0.09, \beta_{\mathrm{PSBw}}^{\mathrm{dp}}=17.9, \Gamma_{\mathrm{PSBw}}^{\mathrm{dp}}=0.09 . \beta_{\mathrm{PSB} \sigma}^{\mathrm{mp}}=518.0$, and $\Gamma_{\mathrm{PSB} \sigma}^{\mathrm{mp}}=$ 1.90. It is noted that for AA2024-T4 the "vein" structure is less well defined than for pure metals and is designated here as a "matrix" structure in recognition of the morphological difference. The relevant $\beta$ and $\Gamma$ parameters, however, are calculated formally from the monopole and dipole building blocks in the same manner as for well-defined vein structures. The contribution to $\beta$ from crack growth is obtained from Eqs.(20)-(22) by assuming $\mathrm{n} \approx 3.0$ (Weertman 1979 ), $\mathrm{B}=1.13$, and $\mathrm{K}_{1 \mathrm{c}} \approx$ $30 \mathrm{MPa} \sqrt{\mathrm{m}}$ (Bucci and Stark, 1996). The parameter A is calculated directly from Eq.(17) and the data tabulated by Weertman (1979) to be approximately $3.8 \times 10^{-11}$ $\mathrm{MPa}^{-3} \mathrm{~m}^{-0.5} \mathrm{cycle}^{-1}$. The elastic nonlinearity parameter $\beta^{\mathrm{e}}$ is determined experimentally to be roughly 4.9 (Cantrell and Yost 1994 ) and $\mathrm{N}_{\text {total }} \approx 3.0 \times 10^{5}$ cycles.

The total nonlinearity parameter $\beta$ of aluminium alloy $2024-\mathrm{T} 4$ for specimens cyclically loaded in uniaxial, stress-control at 276MPa from zero to full load is plotted in Fig.2 as a function of percent fatigue life from the virgin state to fracture. The contribution from substructural evolution is calculated from the data of Grobstein et al. (1991) for stress-controlled loading of polycrystalline nickel at 241MPa and from Eqs.(6), (10), (12), (13), and (25) using the material constants for AA2024-T4. The contributions from crack growth are calculated from Eqs.(20)-(22) and the abovecalculated Paris law parameters for AA2024-T4. The contributions to $\beta$ calculated for the matrix substructure (broken line), the PSBs (solid line), and crack growth (dotted line) are indicated. Also shown in the graph are experimental data points obtained from acoustic harmonic generation measurements previously reported (Cantrell and 
Yost 2001) of ASTM standard 'dogbone' specimens of AA2024-T4 fatigued at a rate of $10 \mathrm{~Hz}$ under uniaxial, stress-controlled cyclic loading at $276 \mathrm{MPa}$ from zero to full load. It is important to point out that large variations in the measured values of $\beta$ occur in the AA2024-T4 experimental data that for a given value of percent life are dependent on the volume of the sample over which the measurements are obtained. Such variations indicate that the fatigue damage is highly localized and that measurements away from the damage site produce smaller values of $\beta$. The values shown in Fig. 2 are the maximum values of $\beta$ obtained for the given values of percent life for which measurements over multiple regions of the specimen gauge section are made.

The agreement between the theoretical curve and the experimental data in Fig.2 is excellent, despite leaning heavily on the substructural evolution data for polycrystalline nickel. Such agreement lends support to the notion that the analytical model may indeed be somewhat generic and thus applicable to wavy slip metals in general with appropriate accounting for different material constants and loading conditions. Such an assumption must be tempered with caution, however, since many of the microstructural input parameters used in the model calculations for both nickel and AA2024-T4 are based on educated estimates from other wavy slip metals that are, as yet, experimentally unconfirmed for the present materials. Further, the experimental measurements of AA2024-T4 are obtained from acoustic harmonic generation data that may involve wave propagation volumes (wave-front crosssectional area times propagation distance) larger than that of material containing relevant fatigue substructures. Hence, the values of the nonlinearity parameter 
measured in such cases would be smaller than the true value. Indeed, as previously reported (Cantrell and Yost 2004) the large variation in the magnitude of the measured nonlinearity parameter from one region to another in the AA2024-T4 specimens suggests that the fatigue damage is highly localized, making the volume of wave interaction an important measurement consideration.

Notwithstanding such uncertainties, the present analytical model predicts and the experimental data confirm that the magnitude of the nonlinearity parameters of AA2024-T4 increase monotonically from the virgin state to fracture as the result of stress-controlled cyclic loading at 276MPa. The contribution from substructural evolution alone produces an increase of more than 250 percent. A measurable contribution from crack growth begins roughly at 80 percent of total fatigue life and rapidly becomes the dominant contribution to $\beta$ as the crack increases in size to fracture.

\section{CONCLUSION}

The model presented here of the dependence of the material (acoustic) nonlinearity parameter on the fraction (percent) of total fatigue life is based on welldocumented microstructural features formed in wavy slip, pure metals subjected to cyclic loading and on the growth of fatigue cracks in the Paris law regime. The model interaction of a stress perturbation (e.g., an acoustic wave) with vein structures and persistent slip bands at a given state of fatigue is based on the accumulated nonlinearities of dislocation monopoles and dipoles that serve as building blocks for the substructures. The calculated values of the nonlinearity parameters for 
polycrystalline nickel above 0.01 percent full life for cyclic loads at $241 \mathrm{MPa}$ and $345 \mathrm{MPa}$ agree to within three percent. The close agreement for such disparate loads suggests that the manner in which the dislocation substructures evolve during fatigue may influence the value of $\beta$ at a given fatigue state more than the specific values of the dislocation densities and volume fractions of the substructures involved. This implies that the model may also be applicable to other materials by accounting appropriately for differences in the fundamental material parameters such as the elastic moduli and Burgers vector, providing that the substructural evolution occurs in a manner that is organizationally and temporally (in terms of percent full life) similar to that of polycrystalline nickel. The successful application of the model to AA2024-T4 using the evolutionary data of polycrystalline nickel appears to substantiate this view.

The effects of crack growth on the nonlinearity parameters are dependent on well-known fracture mechanics concepts and material parameters that are documented for a wide range of materials. The present model predicts that crack dimensions smaller than that of the Paris law regime (less than a radius of $\sim 250 \mu \mathrm{m}$ ) make negligible contributions to the nonlinearity parameter, but cracks in the Paris law regime make significant (even dominant) contributions during the last 10-20 percent of fatigue life. Tong and Bailon (1995) have shown that the plastic zone around the crack tip generates dislocation substructures corresponding to those found in single crystal material cyclically loaded in plastic strain control. Particular substructures occur in well-defined regions in the plastic zone in accordance with the magnitude of the plastic strain experienced by the material in that region. Hence, cell structures, labyrinths, PSBs, veins, and dislocation tangles, respectively, dominate distinct 
regions in the plastic zone as a function of distance from the crack tip. The regions increase in size in accordance with the increase of the plastic zone as the crack length increases during fatigue.

In contrast to pure metals many alloys such as AA2024-T4 commonly contain numerous inclusions and voids ("dirty” material) having proximate cracks. The cracks serve as stress raisers that under cyclic loading produce localized regions of damage, not only from the cracks themselves but also from the dislocation substructures generated in the plastic zone around the crack tip. It is surmised that such localized damage would produce monotonically increasing values of the nonlinearity parameter as a function of decreasing distance from the crack tip. The agreement in Fig. 2 between the maximum measured values of the nonlinearity parameters and the theoretical curve of AA2024-T4 for a given percent full life may thus be the result of the dislocation substructures generated in the plastic zone during crack growth. Indeed, a corresponding substructural evolution would occur even without the presence of pre-fatigue cracks, but it is expected that the evolved substructures would be more globally and uniformly distributed. From a consideration of Fig. 1 for a pure metal and Fig. 2 for a dirty metal it appears that in terms of percent full life expended it makes little difference that the damage is local or global. Near fracture the fatal damage is always local.

The generic character of the model interactions and the relative insensitivity to loading conditions as indicated in Fig. 1 suggest that the present results may apply at least in principle to all wavy slip metals subjected to similar loading conditions, providing appropriate consideration is given to differences in the material constants 
and to modifications in the substructure evolutionary process due, for example, to alloying or heat treatment. The excellent agreement between the theoretical curve and the experimental data for AA2024-T4 lends support to this notion despite the aforementioned uncertainties in the values of several microstructural input parameters used in the model and in the measured nonlinearity parameters resulting from acoustic wave interaction volumes. The present model represents a significant advance in our understanding of the nonlinear interaction of sound waves with fatigue substructures, but further work in experiment and modelling is required. A more comprehensive understanding of wave-microstructure interactions and the assessment of model parameters resulting from such interactions at the most fundamental level must be acquired to gain confidence in model predictions and reliability, as well as to establish the limitations of model and testing applications. Such an understanding is presently being pursued through research with microscopically well-characterized single crystals and polycrystals of fatigued pure metals and metal alloys.

\section{ACKNOWLEGMENT}

I thank Professor L. M. Brown, FRS, of the Cavendish Laboratory, University of Cambridge, for his helpful comments. 


\section{REFERENCES}

Antonopoulos, J. G., Brown, L. M., and Winter, A. T., 1976, Phil. Mag. 34, 549.

Antonopoulos, J. G., and Winter, A. T., 1976, Phil. Mag. 33, 87.

Brown, L. M., 1981, Dislocation Modelling of Physical Systems, edited by M. F. Ashby, R. Bullough, C. S. Hartley, and J. P. Hirth (Pergamon, Oxford), p. 51.

Brown, L. M., 2000, Mater, Sci. Engng A 285, 35.

Brown, L. M., and Ogin, S. L., 1981, Dislocation Modelling of Physical Systems, edited by M. F. Ashby, R. Bullough, C. S. Hartley, and J. P. Hirth (Pergamon, Oxford), p. 501.

Bucci, R. J., and Stark, Jr., E. A., 1996, ASM Handbook, Vol. 19, Fatigue and Fracture (ASM International, Materials Park, OH), pp. 771.

Cantrell, J. H., 2004, Proc. R. Soc. London A 460, 757.

Cantrell, J. H., and Yost, W. T., 1994, Phil. Mag. A 69, 315.

Cantrell, J. H., and Yost, W. T., 2001, International J. Fatigue 23, S487.

Christ, H.-J., 1996, ASM Handbook, Vol. 19, Fatigue and Fracture (ASM International, Materials Park, OH), pp. 73.

Frouin, J., Sathish, S., Matikas, T. E., and Na, J. K., 1999, J. Mat. Res. 14, 1295.

Grobstein, T. L., Sivashankaran, S., Welsch, G., Paigrahi, N., McGervey, J. D., and Blue, J. W., 1991, Mat. Sci. Eng. A 138, 191.

Hertzberg, R. W., 1989, Deformation and Fracture Mechanics of Engineering Materials (Wiley, New York). 
Hull, D. and Bacon, D. J., 1984, Introduction to Dislocations, Pergamon, Oxford.J.

Kratochvil, J., 2001, Mater. Sci. Engng. A 309-310, 331.

Kuhlman-Wilsdorf, D., and Laird, C., 1980, Mater. Sci. Engng, A 46, 209.

Mirshams, R. A., Xiao, C. H., Wang, S. H., and Yin, W. M., 2001, Mater. Sci. Engng. A315, 21.

Mughrabi, H., 1981, Proc. $4^{\text {th }}$ Int. Conf. On Continuum Models of Discrete Systems, edited by O. Brulin and R. K. T. Hsieh (North Holland, Amsterdam), p. 241.

Mughrabi, H., Ackermann, F., and Herz, K., 1978, Fatigue Mechanisms, Proc., ASTM-NBS-NSF Symposium., May 22-24, 1978, Kansas City, MO, Amer. Soc. Testing Mater. Spec. Tech. Publ. 675 (ASTM, Philadelphia) p. 69.

Na, J. K., Cantrell, J. H., and Yost, W. T., 1996, Review of Progress in QNDE, Vol. 15, edited by D. O. Thompson and D. E. Chimenti (Plenum, New York), p. 1347.

Nazarov, V. E. and Sutin, A. M., 1997, J. Acoust. Soc. Am. 102, 3349.

Neumann, P., 1983, Physical Metallurgy, edited by R. W. Cahn and P. Haasen, (Elsevier-Science, New York), p. 1554.

Paris, P. C., and Erdogan, F., 1963, Trans. ASME-J. Basic Engineering 85, 528.

Tong, Z.-X., Bailon, J.-P., 1995, Fatigue Fract. Engng. Mater. Struct. 18, 847.

Wang, R., and Mughrabi, H., 1984, Mater. Sci, Engng. 63, 147.

Wang, R., Mughrabi, H., McGovern, S., and Rapp, M., 1984, Mater. Sci. Engng. 63, 219.

Weertman, J., 1979, Fatigue and Microstructure (American Society for Metals, Metals Park), p. 279. 
Winter, A. T., 1974, Phil. Mag. 30, 719. 


\section{FIGURE CAPTIONS}

Fig. 1. Graph of calculated material (acoustic) nonlinearity parameter plotted as a function of percent total fatigue life for polycrystalline nickel cyclically loaded in stress control at $345 \mathrm{MPa}$ (mostly upper curve) and at $241 \mathrm{MPa}$ (mostly lower curve).

Fig. 2. Graph of material (acoustic) nonlinearity parameter plotted as a function of percent total fatigue life for aluminium alloy 2024-T4. The continuous curve is calculated from the analytical model. The filled circles with error bars are experimental data points obtained from acoustic harmonic generation measurements (Cantrell and Yost 2001). 


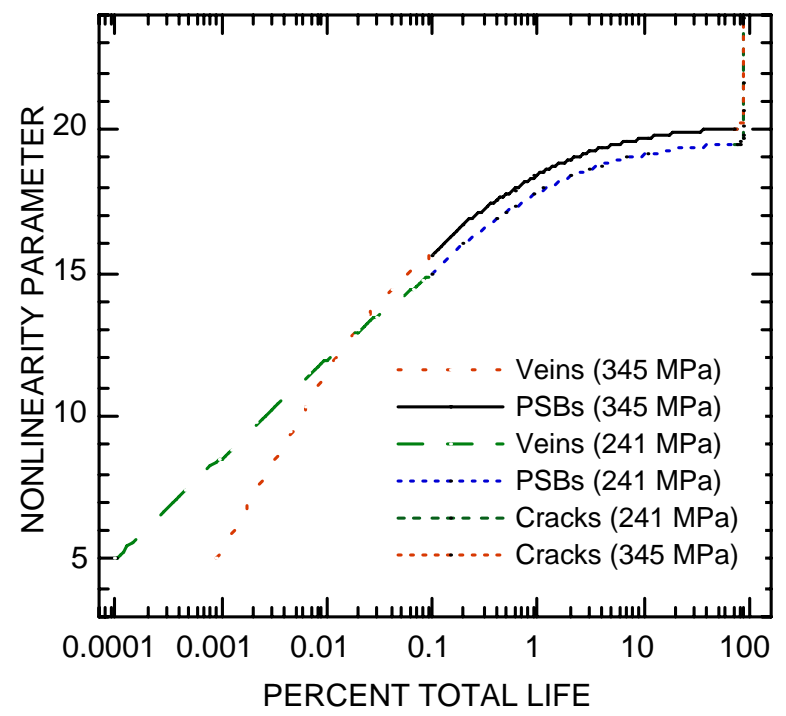

Figure 1. Cantrell 


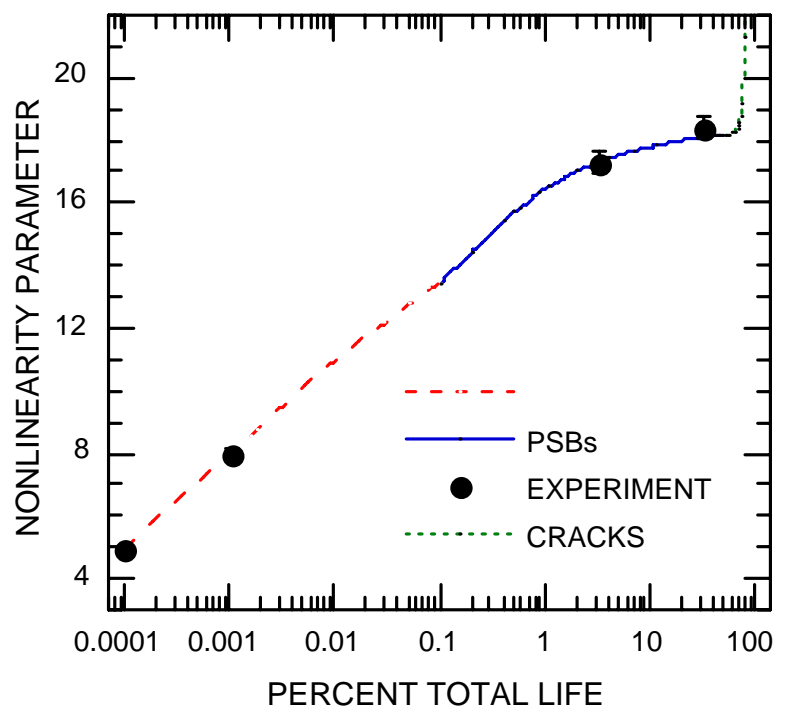

Figure 2. Cantrell 